\title{
A Fibril Structure Model for Stellar Prominences
}

\author{
R. Oliver and J. L. Ballester \\ Departament de Física, Universitat de les Illes Balears, E-07071 Palma \\ de Mallorca, Spain
}

\begin{abstract}
Extensive observational background exists about the presence of cool material clouds embedded in the stellar coronae of rapidly rotating, late-type stars. Observations of such clouds in HKAqr (Gliese 890 ) and HD197890 suggest that the clouds are at heights smaller than the star's corotation radius and could be looked at as a phenomenon similar to that of solar prominences. Recent observations of solar prominences have reinforced the evidence about their fibril structure made of many long, thin magnetic flux tubes making angles about 25 degrees with the direction of the filament channel, with only the central $10-20 \%$ of the magnetic flux tubes filled with cool matter, which can produce a depression at the summits of the flux tubes. Then, assuming a similar structure for the stellar cool clouds, we have looked for the physical characteristics of such stellar prominences, i.e. the size of the flux tube depression, the density, temperature, half-width and supported mass of the cool region, taking into account gravity variation with height and centrifugal acceleration, since such clouds have been detected at great heights within stellar coronae belonging to rapid rotators.
\end{abstract}

\section{Introduction}

Recently, claims about the discovery of cool, prominence-like material embedded in hot stellar coronae of late-type stars have been made. Collier-Cameron and Robinson (1989a, b), using spectra of the $\mathrm{H} \alpha$ profile in the active, rapid rotator $\mathrm{K0}$ dwarf $\mathrm{AB}$ Doradus, suggested that such features originate in cool, dense clouds embedded in and corotating with the hot corona.The observations indicate that the clouds form in a region just outside the Keplerian corotation radius having a projected extension between 3 and $20 \%$ of the stellar surface area. The temperatures are in the range $4500-14,000 \mathrm{~K}$ and densities could be between $10^{9} \mathrm{~cm}^{-3}$ and $10^{13} \mathrm{~cm}^{-3}$. The clouds seem to be forced to corotate with the star, thus indicating the presence of closed magnetic loops reaching great heights in the stellar atmosphere. Doyle and Collier-Cameron (1990) made observations of the $M$ dwarf Gliese 890 , concluding that the spectral features were consistent with the existence of a cloud of neutral hydrogen with a projected area of 0.25-0.40 times that of the stellar disc. In this case, the cloud seems to be close to but below the corotation radius. Collier-Cameron and Woods (1992) found evidence for prominence-like circumstellar material in four rapidly rotating $\mathrm{G}-\mathrm{K}$ stars of the $\alpha$ Persei cluster. In this case, the observations suggest that the clouds lie preferentially outside the Keplerian corotation radius. Jeffries 
(1993) obtained $\mathrm{H} \alpha$ spectra of the rapid rotator HD197890. The behaviour of the absoption transients is consistent with the model of cool clouds of material trapped in corotating, closed magnetic structures above the star's surface. Also, it seems that some of the clouds are located outside the Keplerian corotation radius while others can be located within the corotation radius. Byrne et al. (1996) and Byrne (1996) have reported the discovery of cool, prominence-like material embedded in the corona of the rapid rotator HKAqr (Gl890). The observations suggest that such features form well below the co-rotation radius and that their dimensions lie between 3 and 20 times those of solar prominences. Further evidence has been obtained by Byrne et al. (1997) who have used visibility plots to show that most of the clouds are located near the equatorial plane, below the corotation radius.

From the theoretical point of view, van den Oord (1988) used a current line model to investigate the possibilities of filament support in binary stars. CollierCameron (1988) studied the properties of coronal loop structures with lengths comparable to or greater than the stellar radius and the classes of solutions, hot and cool, which can be found. He suggested that an explanation for neutral hydrogen clouds is that they can be formed by thermal collapse of large Xray loop structures, whose summits extend beyond the Keplerian corotation radius, with centrifugal acceleration providing support against the RayleighTaylor instability. Ferreira and Jardine (1995) have presented a model, using a massive circular line current, describing the equilibrium of a stellar filament in the equatorial plane of a rapidly rotating, late-type star. Recently, Byrne et al. (1997) have studied the equilibrium of prominence-like clouds in latetype rapid rotators, presenting arguments with respect to the nature of the clouds, i.e. whether they are filamentary-like structures or coronal loop-like structures. They conclude that the filamentary hypothesis can be ruled out since filaments can only have stable equilibria up to 0.63 times the corotation radius and most of the detected clouds are located at larger heights. Then, an interesting possibility is that the clouds are of coronal loop-like nature with a temperature inversion near the apex. Van den Oord and Zuccarello (1996) showed that, below the corotation radius, such temperature reversals can be created when there is insufficient heating near the apex of the loops or when the cross-section increases towards the apex.

\section{Basic Model}

Observations of quiescent solar prominences have shown very fine structures in the body of the filament, suggesting they are composed of small-scale structure aligned with the magnetic field and anchored at many different foot-points. Such fibrils have been well-resolved by $\mathrm{Yi}$ et al. (1991) and Yi and Engvold (1991) in their observations of the HeI $\lambda 10830 \AA$ in quiescent filaments. From such observations these authors conclude that the prominence matter is contained in many long, thin magnetic flux tubes making angles of about 25 degrees with the direction of the filament channel. Also, it seems that only the central $10-20 \%$ of the magnetic flux tubes is filled with cool matter.

Ballester and Priest (1989) developed a model to explain the observed fibril structure of solar prominences. They assumed that the fibril structure of a 
prominence is composed of flux tubes containing hot plasma $\left(T_{i} \sim T_{c}\right)$ over most of their length and cool plasma $\left(T_{i} \ll T_{c}\right)$ near their summits representing the cool prominence body. They start with a hot flux tube and assume that a cool condensation appears near the top of the flux tube, which produces a downward anti-buoyancy force which must be balanced by other forces acting on the flux tube. The magnetic field is assumed to be in equilibrium and the plasma is in a state of hydrostatic equilibrium along the magnetic field lines.

This model was further developed by Degenhardt and Deinzer (1993) by including a simplified energy equation without a conduction term. Schmitt and Degenhardt (1998) extended this model by solving, numerically, the full energy equation together with the equilibrium equations, which improves the temperature gradient between the prominence and the corona, without modifying substantially the obtained values for the rest of parameters (density, temperature, size of the depression) characterizing the prominence region.

Here, we assume that cool clouds, observed below the corotation radius in late-type stars, are stellar prominences having a fibril structure like solar ones, and our aim is to obtain the physical characteristics (density, temperature, size of the depression, half-width, supported mass) of the cool part of such fibril structure. To this end, we have adapted the model by Degenhardt and Deinzer (1993) to the stellar case by taking into account gravity variation with height and centrifugal acceleration, since such clouds have been detected at great heights within stellar coronae belonging to rapid rotators.

\section{Rapid rotators: HKAqr (Gl890) and HD197890}

Cool clouds have been observed in these stars with the common feature that they seem to be located below the corotation radius. HKAqr is a dM2Ve star with a radius about $0.56 R_{\odot}$, mass about $0.4 M_{\odot}$ and a rotation period of 10.34 hr. HD197890 is a $\mathrm{KOV}$ star with a radius about $0.85 R_{\odot}$, mass about $0.75 M_{\odot}$ and a rotation period of $8.04 \mathrm{hr}$. Since the physical characteristics and rotation periods are different, we have performed separate calculations for HKAqr and HD197890.

To know the influence of different parameters on prominence characteristics, we have varied the coronal temperature, the heating constant, the coronal base pressure and the critical coronal height. In all cases, the values of three parameters have been kept constant and we have modified the fourth one.

The most important parameter affecting prominence characteristics is the height of flux tube summit, since when it is increased the size of the dip grows spectacularly. On the other hand, the rest of parameters are still consistent with the existence of cool material at the dip.

We have also studied, for a solar-type star, the influence of the centrifugal acceleration on prominence characteristics. To this end, we have varied the angular velocity between one and a hundred times the solar value, finding that the increase of the angular velocity does not substantially affect prominence characteristics.

Two important quantities to be determined are the mass in the cool region and the half-width of the cool region. The half-width of the cool region has been taken as the horizontal distance from the dip position to the point where the 
internal pressure becomes equal to the central pressure divided by e. Once we know the height at which the above condition is matched, we can compute the contained mass. These calculations have been done for HKAqr and HD 197890 and the obtained mass is similar to the mass of a well developed quiescent solar prominence. Assuming a cool cloud $800,000 \mathrm{~km}$ long, 30,000 km high and 20,000 $\mathrm{km}$ wide, the mass is about $10^{13} \mathrm{~kg}$.

In summary, this fibril structure model for stellar prominences located below the corotation radius indicates that the most important parameter affecting prominence characteristics is the height of the flux tube summit while the angular velocity has no importance. The critical coronal height determines the depth of the depression, where the cool material is located, influencing, at the same time, the half-width of the prominence and the amount of mass contained within it.

\section{References}

Ballester, J. L. and Priest, E. R. 1989, A\&A, 225, 213

Byrne, P.B. 1996, in Magnetodynamic Phenomena in the Solar Atmosphere, Y. Uchida, T. Kosugi and H. S. Hudson (eds.), Kluwer Acad. Publ., Dordrecht, Holland, p. 139

Byrne, P.B., Eibe, M.T. and Rolleston, W.R.J. 1996, A\&A, 311, 651

Byrne, P.B., Eibe, M.T. and van den Oord, G. H. J. 1997, in preparation

Collier-Cameron, A. 1988, MNRAS, 233, 235

Collier-Cameron, A. and Robinson, R.D. 1989a, MNRAS, 236,57

Collier-Cameron, A. and Robinson, R.D. 1989b, MNRAS, 238, 657

Collier-Cameron, A. and Woods, J.A.. 1992, MNRAS, 258, 360

Degenhardt, U. and Deinzer, W. 1993, A\&A, 278, 288.

Doyle, J.G. and Collier-Cameron, A. 1990, MNRAS, 244, 291

Ferreira, J.M.T.S. and Jardine, M. 1995, A\&A, 298, 172

Jeffries, R.D. 1993, MNRAS, 262, 369

Schmit, D. and Degenhardt, U. 1998, in G. Klare (ed.) Rev. Mod. Astron., Springer-Verlag, Berlin (in press).

van den Oord, G.H.J. 1988, A\&A, 205, 167

van den Oord and G.H.J., Zuccarello, F. 1996, in Stellar Surface Structure, Strassmeier, K. G. and Linsky, J. L. (eds.), Kluwer Acad. Publ., Dordrecht, Holland, p. 433

Yi, Z., Engvold, O. and Keil, S. L. 1991, Solar Phys, 132, 63

Yi, Z. and Engvold, O. 1991, Solar Phys, 134, 275 